\title{
Grupos interactivos: estrategia para la mejora de la convivencia, la participación y el aprendizaje
}

\author{
Interactive groups: a strategy to improve \\ coexistence, participation and learning \\ Edurne CHOCARRO DE LUIS y Magdalena SÁENZ DE JUBERA OCÓN \\ Universidad de La Rioja
}

Recibido: Octubre 2014

Evaluado: Enero 2015

Aceptado: Febrero 2015

\begin{abstract}
Resumen
Los grupos interactivos son un modo de trabajo en el aula que se aprovecha de los beneficios del aprendizaje dialógico, de las interacciones entre las personas y para ello, el alumnado se agrupa de modo heterogéneo bajo la tutela de una persona adulta, cuya responsabilidad es garantizar la participación de todos sus miembros. Esta actuación se perfila como una evidencia de éxito propia de las escuelas inclusivas convertidas en comunidades de aprendizaje. Su puesta en escena requiere de un tiempo para asimilar sus principios y, por ello, el objetivo de este trabajo es presentar las dificultades así como las ventajas encontradas a partir de la experiencia incipiente del Colegio Caballero de La Rosa de Logroño. La metodología utilizada ha sido la investigación acción participativa al contar con los distintos agentes partícipes: familiares, profesores y alumnado en dos focus groups llevados a cabo al inicio y fin del curso 2012/2013 con objeto de contrastar las primeras impresiones con aquellas más asentadas tras un periodo de rodaje y así extraer resultados desde la práctica.

Los resultados se organizan en torno a tres ámbitos impulsados en las comunidades de aprendizaje: académico, convivencial y participativo. Se evidencia que los grupos interactivos han logrado una mayor implicación del alumnado motivados por la dinámica de la misma y que la colaboración de familiares y agentes externos así como los vínculos establecidos entre todos los implicados revierten en los resultados de aprendizaje y mejoran la convivencia.

Lejos de intrusismo, la posibilidad de contar con más personas en el aula ofrece lentes nuevas que pueden aportar mejoras en la convivencia y dar más luz al aprendizaje de los alumnos.
\end{abstract}

Palabras clave: aprendizaje, interacción y participación.

\begin{abstract}
Interactive groups are a way of classroom work that takes advantage of the benefits of dialogic learning, of interactions between people, and for that, students are grouped heterogeneously under the tutelage of an adult, whose responsibility is to ensure the participation of all its members. It is a performance that is emerging as an evidence of successful inclusive schools turned into learning communities. Its staging requires time to assimilate its principles and therefore the aim of this paper is to present the difficulties as well as the advantages collected
\end{abstract}


from the incipient experience at the Caballero de La Rosa school, in Logroño, La Rioja. The methodology used was participative action research by having a diversity of participant agents: family, teachers and students in two focus groups conducted at the beginning and at the end of the school year 2012/2013. The aim was to contrast the first impressions with those settled after a period of development and thus to obtain results from practice. The results are organized around three areas driven in learning communities: academic, coexistential and participative. It shows that interactive groups have achieved a greater student involvement motivated by its own dynamics. It also shows that family and external agents' collaboration, as well as the links among them, reverts to learning outcomes and help to improve coexistence. Far from being considered an intrusion, the possibility of counting on more people in the classroom offers new lenses that can provide improvements in the coexistence and give more light to student learning.

Keywords: learning, interaction and participation.

Existen proyectos educativos sustentados en la convicción de que la participación de las familias y agentes externos de la comunidad influye en el rendimiento escolar del alumnado, mejorando sus resultados de aprendizaje e impulsando una educación inclusiva (INCLUD-ED, 2009). Esta se materializa con la puesta en práctica de cuatro elementos que se resumen en que: la inclusión es un proceso; busca la presencia, participación y él éxito de los estudiantes; precisa la identificación y eliminación de barreras y enfatiza al alumnado en riesgo de marginalización, exclusión o fracaso escolar (Echeita y Ainscow, 2011, p. 32-34). Un proyecto educativo que recoge estos cuatros elementos son las llamadas Comunidades de Aprendizaje (Racionero y Serradell 2005; Flecha y Ortega, 2012). Según Elboj, Puigdellívol, Soler y Valls (2005) se definen como:

un proceso de transformación social y cultural de un centro educativo y de su entorno para conseguir una sociedad de la información para todas las personas, basada en el aprendizaje dialógico, mediante una educación participativa de la comunidad que se concentra en todos sus espacios, incluida el aula. (p.74)

En España se han instituido distintas redes para impulsarlo como la Red Andaluza de Comunidades de Aprendizaje. El reto de convertirse en Comunidad de Aprendizaje exige un proceso de transformación secuenciado en una serie de fases, de exigido cumplimiento donde la voz de la familia es clave en su desarrollo. Esto es, cuando un centro educativo apuesta por ello, la familia participa en toda su amplitud. Así, está invitada a asistir a las sesiones de formación propias del primer paso denominado sensibilización con el objeto de acercar los principios y valores de la propuesta a la comunidad educativa. Tras la fase de sensibilización, al menos el $90 \%$ del profesorado debe estar de acuerdo en llevar a cabo el proyecto, liderados por el equipo directo, y también es necesario que los padres y madres aprueben mayoritariamente la conversión en Comunidad de Aprendizaje, de este modo todos los agentes partícipes tienen oportunidad de aportar sus ideales de escuela en la denominada fase de sueño. Hechos explícitos éstos, se establecen cuáles son los prioritarios para una mejora de la escuela y se organizan comisiones mixtas para su logro en base a las temáticas de trabajo en las que, nuevamente, la familia también tiene voz. En definitiva, en estos 
modelos de escuela, la relación familia y escuela es algo más que intercambio de información y, como avala el proyecto INCLUD-ED (2009) responde a las premisas de una participación educativa, galante de propiciar una escuela inclusiva pues incide en contar con ellos tanto en la dinámica interna de funcionamiento y enseñanza como en los procesos de formación, como ocurre en las comunidades de aprendizaje. Además, su participación es fundamental en las dinámicas de aula que se generan en favor de escenarios participativos inclusivos (Elboj, Puigdellivol, Soler y Valls, 2005) como los grupos interactivos (Racionero y Valls, 2007), una evidencia de éxito que consiste en dividir el aula en grupos de trabajo heterogéneos de cuatro o cinco alumnos, con la particularidad de la presencia de un adulto externo por grupo que anima y dinamiza la participación de sus miembros durante el desarrollo de la actividad prevista. Basándose en el poder de las interacciones, su cometido es cerciorarse de que todos participan en la elaboración de la tarea conjuntamente refrendados por el valor del aprendizaje dialógico (Aubert, Flecha, García, Flecha y Racionero, 2008). Cada grupo, por tanto, realiza una actividad diferente planteada por el profesor que tendrán que resolver entre todos los miembros en un tiempo determinado y, vencido éste, los alumnos rotarán a la siguiente actividad, tantas como grupos haya, de tal modo que al finalizar la sesión, todo el alumnado habrá realizado todas las actividades planteadas. Los voluntarios se mantienen en el grupo dispensando el material y gestionando sus interacciones. Estos voluntarios pueden ser familiares, padres, vecinos de la comunidad, etc. Al aumentar las interacciones se acelera el aprendizaje (Elboj, Puigdellivol, Soler y Valls, 2002).No es necesario que dominen el tema, el profesor se encargará de resolver dudas si fuera necesario quien, a su vez, controla tiempos y vigila la dinámica además de planificar las actividades (Flecha y Ortega, 2012; Flecha y Soler, 2013).

Insertos en este marco, los objetivos de la siguiente investigación fueron: a) analizar las impresiones de padres, profesorado y alumnado sobre el alcance de los grupos interactivos como metodología inclusiva en las escuelas convertidas a Comunidades de Aprendizaje y b) refrendar sus impresiones antes y después de la puesta en marcha de los grupos interactivos para corroborar sus expectativas al respecto. Se espera encontrar que los grupos interactivos, sobre todo, repercutan en una mejora del clima social del colegio mejorando la convivencia, aceleren la implicación por aprender y, además, sea una estrategia óptima para garantizar la atención a la diversidad. Además nos guía la hipótesis que el alumnado se sentirá más partícipe si es consciente también de la implicación de la familia en el centro educativo.

\section{Método}

El diseño de esta investigación se corresponde con la denominada investigación acción participativa. Un diseño al alza que involucra a todos los responsables o partícipes, de uno u otro modo, en el tema de estudio durante el mismo. Se analiza una misma realidad desde distintas perspectivas dando respuesta a intereses comunes. Como explican Gallardo y Camacho (2008) no existe una definición consensuada pero ellos se decantan por describirla como: 
Una estrategia metodológica de investigación en relación directa con la formación, la participación, el desarrollo comunitario y la transformación social. Tendente a integrar el conocimiento científico y popular para propiciar el cambio social a fin de mejorar las condiciones y calidad de vida de las personas y colectivos de una determinada comunidad. (p.30)

Tanto los objetivos como la naturaleza de la investigación se prestan a este tipo de investigaciones pues su planteamiento pretende la implicación de la comunidad que, a su vez, son los principales beneficiarios tanto del propio desarrollo del proyecto como de los resultados alcanzados (Salazar, 1992; López de Ceballos, 1998; Gallardo y Camacho, 2008).

Así pues, el papel de experto y participante se entremezclan y se diluyen en el desarrollo de la propia investigación pues todas las voces son consideradas en igualdad de condiciones, principio clave del aprendizaje dialógico (Aubert, Flecha, García, Flecha y Racionero, 2008).

\section{Instrumento}

Se consideró oportuno utilizar el grupo de discusión (Krueger, 1991) como herramienta de recogida de información y así concentrar las impresiones de los tres agentes principales del proceso estudiado en un mismo escenario. Cada uno ofrece una mirada distinta aportando nuevas perspectivas, dando luz a nuestros objetivos de estudio. En este caso, se realizaron grupos de discusión, uno tras la experiencia piloto con grupos interactivos con objeto de recoger las primeras impresiones sobre esta metodología en cuanto a expectativas y otro al fin del primer curso para cotejarlas y comprobar los resultados alcanzados, de acuerdo al segundo objetivo de esta investigación. Para llevarlo a cabo se elaboró cuidadosamente un guión con las cuestiones formuladas a los participantes, con el fin de ajustarse perfectamente a los intereses del estudio (Krueger y Casey, 2000).

A continuación se presentan las preguntas ordenadas conforme al momento en el que se presentaron durante el debate. Esto es, el grupo de discusión se iniciará formulando preguntas para "romper el hielo" y animar la conversación, seguidamente se lanzará alguna cuestión introductoria sobre el tema que permita encuadrarlo y sucederán otras más concisas con objeto de orientarlo hacia las preguntas clave del estudio. Finalmente, se cerrará la discusión con una pregunta a modo de conclusión final o valoración global de los temas tratados.

Considerando estas orientaciones, se propusieron las preguntas semiestructuradas que se detallan en la tabla 1. 


\begin{tabular}{|c|c|}
\hline $\begin{array}{l}\text { Abrir } \\
\text { diálogo }\end{array}$ & $\begin{array}{l}\text { 1. Presentación de los participantes especificando nombre y } \\
\text { rol que representa (profesor, padre, alumno, voluntario...) }\end{array}$ \\
\hline Introductoria & $\begin{array}{l}\text { 2. ¿Por qué aprobasteis implantar el proyecto de Comunidades } \\
\text { de Aprendizaje? }\end{array}$ \\
\hline \multirow[t]{2}{*}{ Transición } & $\begin{array}{l}\text { 3. ¿Cuál es vuestra opinión sobre que las puertas del centro } \\
\text { estén abiertas a padres y personas voluntarias, es decir, que se } \\
\text { permita su participación en el proceso de enseñanza- } \\
\text { aprendizaje de los alumnos? }\end{array}$ \\
\hline & $\begin{array}{l}\text { 4. ¿Creéis que la puesta en marcha de los grupos mejora el } \\
\text { rendimiento académico de los alumnos? }\end{array}$ \\
\hline $\begin{array}{l}\text { Cuestiones } \\
\text { claves }\end{array}$ & $\begin{array}{l}\text { 5. ¿Pensáis que también mejora la convivencia el centro } \\
\text { educativo? }\end{array}$ \\
\hline & $\begin{array}{l}\text { 6. ¿En qué ha repercutido la participación de agentes externos? } \\
\text { 7. ¿Os habéis encontrado alguna dificultad a la hora de hacer } \\
\text { posible este tipo de participación? ¿Cuáles? }\end{array}$ \\
\hline $\begin{array}{l}\text { Valoración } \\
\text { final }\end{array}$ & 8. Para concluir, ¿Añadiríais algo a las reflexiones realizadas? \\
\hline
\end{tabular}

Tabla 1. Preguntas propuestas al grupo de discusión.

El guión estuvo sometido a una serie de modificaciones al hilo de las aportaciones de los participantes.

\section{Participantes}

Según Llopis (2004, p.100): “los grupos de discusión se componen de entre siete a diez participantes, lo que puede considerarse como límite mínimo y máximo, dentro de los cuales un grupo puede funcionar correctamente". Es preferible que el grupo no sea numeroso para evitar subdivisiones. En este sentido, puede parecer también que sea aconsejable que los participantes no tengan mucha relación entre sí para evitar conversaciones paralelas al tema de estudio.

En este caso, el grupo de discusión, en ambas ocasiones, se constituyó por tres profesores (tutor y Maestra de Educación Física -a la vez coordinadora de grupos interactivos-, jefe de estudios), tres voluntarios (tres familiares), y tres alumnos/as de sexto de primaria, tal y como se refleja en la tabla 2. Los nueve integrantes fueron seleccionados por el equipo directivo del centro pues les constataba su participación, entusiasmo y deseo por poner en marcha el proyecto. 


\begin{tabular}{cc}
\hline Código & Rol \\
\hline M1 & Tutor. Curso 5 Educación Primaria. \\
Je & Jefe de Estudios. Maestro especialista en Educación Musical. \\
M2 & Maestra Especialista en Educación Física. Coordinadora grupos \\
& interactivos. \\
A1 & Alumno Sexto de Primaria. \\
A2 & Alumno Sexto de Primaria. \\
A3 & Alumna Sexto de Primaria. \\
F1 & Padre alumno Educación Primaria. \\
F2 & Padre alumno Educación Primaria. \\
\hline F3 & Madre alumno Educación Primaria. \\
\hline
\end{tabular}

Tabla 2. Miembros del grupo de discusión y códigos asignados.

Todos los miembros comparten inquietudes comunes emanadas de su interés por apoyar el proceso de transformación hacia comunidad de aprendizaje en el Colegio Caballero de la Rosa de Logroño que apostó por convertirse en tal modelo educativo en marzo de 2012, con carácter pionero en La Rioja. La heterogeneidad de su alumnado, el alto índice de población inmigrante, sobre todo pakistaníes, así como la falta de colaboración de las familias por la dificultad del lenguaje lo motivaron. Alcanzado el consentimiento de la comunidad educativa, se comenzó por introducir evidencias de éxito en los procesos de enseñanza como los grupos interactivos y tertulias dialógicas como modelos de actuaciones inclusivas (Flecha y Ortega, 2012). Así, en Mayo y Junio de ese curso realizaron una experiencia piloto con grupos interactivos en los cursos de quinto y sexto de educación primaria para familiarizarse con la dinámica y también anticipar posibles barreras con vistas a su plena puesta en marcha en el siguiente curso. Estos alumnos trabajaban mediante grupos interactivos dos horas a la semana, una hora en matemáticas y otra en lenguaje. No obstante, se consideró oportuno plantear un grupo de discusión para conocer las primeras impresiones de los principales protagonistas: alumnos, profesores y miembros de la comunidad, que se celebró en Octubre de 2012 a modo de termómetro para medir sus expectativas iniciales y valoraciones para encauzar su puesta en escena en el curso 2012/2013, pero ya en todos los cursos de primaria e infantil. Esta discusión sirvió de espejo para ver reflejado tanto los logros como posibles puntos a mejorar que son complicados de percibir inmersos en la dinámica. No obstante, trascurrido el curso, se optó por repetir el grupo de discusión para refrendar el cumplimiento de esas expectativas, esto es, valorar el grado de realización de esos deseos o primeras intuiciones.

\section{Análisis de los datos}

Tras llevar a cabo el grupo de discusión, se transcribió el diálogo y se analizó empleando la técnica de análisis de contenido. Para Martínez (2007, p. 94) “el análisis de contenido consiste básicamente en categorizar y codificar los datos en función de 
criterios que se establecen para analizar e interpretar adecuadamente los significados de los comentarios emitidos por los sujetos”.

Sin embargo, las preguntas lanzadas en el grupo de discusión limitan las categorías, aunque previamente estas preguntas fueron consensuadas con el equipo directivo. Las investigadoras abordaron el análisis teniendo presentes tres temas principales: académico, convivencial y participativo como ámbitos impulsados en las comunidades de aprendizaje.

Teniendo en cuenta estos tres temas se estableció el sistema de categorías y tras finalizar este proceso y, como estrategia encaminada a la validez del estudio, ambas investigadoras lo contrastaron a modo de revisión por pares (Creswell y Miller, 2000; Suárez, del Moral y González, 2013). En el ámbito académico se consideró la implicación y motivación en los procesos de aprendizaje así como la organización de los grupos; en el convivencial se atendió al clima de aula y el ámbito participativo se analizó desde dos puntos de vista: por un lado, desde las relaciones del centro con la familia, con la comunidad y con la administración educativa y, por otro, desde su contribución a la creación de una escuela inclusiva.

A partir de un acuerdo consensuado, se trabajó conjuntamente para reagrupar la información en estos temas y categorías teniendo presente también la opinión del centro en todo el proceso. El análisis de la información es un proceso continuo y cíclico dado que se produce una constante interacción entre la recogida y la disposición de los datos y la extracción y verificación de las conclusiones durante todo el proceso de la investigación.

\section{Resultados}

a) Ámbito académico

El principal objetivo de este ámbito se centra en la mejora de los resultados académicos para todo el alumnado. No obstante, en este caso, el profesorado manifiesta que tras un año de transformación en Comunidad de Aprendizaje sus prioridades no son tanto valorar los logros académicos sino la implicación del alumnado y su motivación con el aprendizaje, categoría objeto de análisis.

En el grupo de discusión inicial los alumnos respondieron positivamente ante las nuevas fórmulas de trabajo. Era poco el tiempo de funcionamiento, apenas un mes, pero ellos ya intuían ciertos beneficios al trabajar en grupos interactivos. Los comentarios literales fueron los siguientes:

"Al hacerlo en grupo se tarda un poco más porque entre que nos pasamos las hojas, tenemos que cambiarnos los lápices.”(A1)

"Creo que disfrutamos porque lo que se aprende no solo hay que dejarlo para sí mismo, hay que compartirlo con los demás.”(A2) 
"A veces hay algo bueno, te los sabes pero ya hace mucho que no lo has utilizado, y ya, de repente, un compañero te lo pregunta, pues tú se lo vas explicando y mientras te vas acordando tú."(A3)

Cabe mencionar que el primer comentario es relevante ya que, entre líneas, puede leerse que todavía debían asentar este todavía nuevo modo de trabajo y requerían de más experiencia para poner en marcha el engranaje con cierta soltura. Ciertos aspectos organizativos fueron subsanándose con el transcurrir de las clases ya que eran muchas las dudas sobre el material más eficaz y su disposición en los grupos. En la experiencia piloto comprobaron que era preferible que los grupos dispusieran solo de una hoja de ejercicios o ficha en vez de que cada miembro utilizase la suya propia, pues así no colaboran y la realizan a modo personal sin interaccionar. Así también, se optó por contar con un solo lápiz por grupo ya que si los alumnos rotaban con sus estuches, solo se preocupaban por no olvidarlo e incluso les servía para atrincherarse y llevar a cabo el ejercicio sin contar con los otros como nos relataba el jefe de estudios. Estos aspectos no son contemplados en la literatura pero resultan de interés didáctico para profesores en ejercicio.

Una profesora explicaba que los alumnos realizan actividades similares a las que podrían hacer en una clase estándar, pero ejecutan un mayor número de las que son capaces de hacer en un período lectivo "tradicional". De hecho, es habitual que alumnos y alumnas desmotivados académicamente disfruten mucho en los grupos interactivos, aunque los contenidos sean prácticamente iguales a los de una clase normal. Lo escuchamos en las palabras de una profesora:

Al final todos los críos realizan todas las actividades, yo en clase con la fórmula tradicional hubiese sido imposible en una hora, pero imposible, pero de este otro modo, es, empírico hacer todo lo que yo planteo a los alumnos. Entonces aprendizaje divertido más rotatorio diríamos en esta fórmula pues es una fórmula sencilla pero que tiene un potencial fantástico.”(M1)

Retomando las aportaciones de los alumnos, en el segundo encuentro de discusión, éstos reforzaron sus cábalas iniciales:

"Algunos alumnos hemos mejorado gracias a grupos interactivos porque en las clases normales igual no estamos tan atentos pero entonces en grupos, pues si lo estamos, porque somos menos y entonces mejoran."(A1)

"Los padres voluntarios y todas las personas que vienen nos enseñanza cosas que ya hemos dado pero se nos habían olvidado."(A2)

“Nosotros también aprendemos cuando corregimos a otros compañeros.”(A3)

Asimismo, los profesores constataron que tras un año de funcionamiento de los grupos interactivos, los participantes en los mismos han asumido su rol y la dinámica de organización y funcionamiento, aunque falta pulir las responsabilidades de cada uno, como explicaba una profesora:

"Les costó meterse en la dinámica de los grupos, pero con el tiempo han ido adquiriendo hábitos de trabajo grupal, son menos individualistas, e incluso 
algunas veces anteponen el trabajo del grupo al suyo propio están muy interesados en que nadie se quede atrás. Además hemos mejorado el interés por la tarea, la atención y la concentración."(M1)

Como cualquier cambio, se requiere de tiempo para asimilar roles y asumir la dinámica y modos de trabajo por ello se mencionó que se requería de cierto calentamiento o entrenamiento para lograr su buen funcionamiento.

\section{b) Ámbito convivencial}

El objetivo de este ámbito es impulsar la mejora del clima en el aula. En el primer grupo de discusión, no se advirtieron avances pues era poco el tiempo invertido en este modo de trabajo, recordamos que fueron unas sesiones a modo experimental, y sobre todo les preocupaba analizar la organización y estructura de los grupos aunque ya había indicios de un cambio en las relaciones de aula como relataba una de las maestras:

"Entonces yo creo que también aquí hay que ver un poco que a veces cuando se dice: ¿y esto va a servir? Estamos pensando en si las notas van a subir, pero ya independientemente de esas mediciones que se puedan hacer pasado un tiempo, ya hay unos resultados del clima del aprendizaje que son fundamentales."(M1)

Añadir que de los testimonios expuestos en el apartado anterior se inducen mejoras en la convivencia que, principalmente, fueron constadas en el segundo grupo de discusión. Así el jefe de estudios y un profesor resaltaron:

"Nos hemos dado cuenta de que muchos de nuestros alumnos no eran conflictivos y después fracasaban en los estudios, sino que, fallaban académicamente y entonces creaban conflictos en las aulas. Existe un mayor respeto entre los alumnos, empatía.... "(Je)

"Había gente que decía y ¿qué resultados hay? Y sí que vamos viendo resultados, sobre todo a nivel de convivencia, cambios de actitudes de chavales, Esta claro que, si un compañero te ayuda a resolver un problema de contenido, de conocimientos, pues evidentemente surge un agradecimiento y en el momento, por ejemplo, del patio, puede aparecer una relación entre ellos porque se han echado un cable. Eso es así, se vive y se agradece."(M2)

De las relaciones entre los miembros de la comunidad educativa emanadas de las opiniones recogidas de los miembros del grupo de discusión nos ocuparemos en el ámbito participativo, porque si bien podría ser objeto de análisis en el ámbito convivencial la mejora de las mismas se deriva directamente del aumento de la participación de los distintos agentes en el centro educativo.

\section{c) Ámbito participativo}

Este ámbito se refiere a la repercusión que el aumento de la participación de la comunidad educativa en el centro tiene en lo relativo al funcionamiento y 
organización escolar como al desarrollo curricular. Así, la presencia de familiares y voluntarios en las aulas ha traído consigo, por un lado, la mejora de las relaciones entre ellos y, por otro, una mayor atención a la diversidad del alumnado, categorías objeto de análisis.

En el primer grupo de discusión, se expuso que la participación de voluntarios importaba otros modelos de trabajo para los alumnos y no tanto mejora en las relaciones personales y con el centro, puesto que el escaso tiempo de desarrollo de los grupos interactivos no lo permitía todavía. No obstante, el alumnado se exponía a personas con distintos hábitos, formas de ser que aportaban actitudes y nuevos conocimientos al aula, tal y como explican maestros y alumnos:

“Algunos son más serios, otros más divertidos.”(A2)

"Yo creo que es mejor ir cambiando de moderador porque, no sé, así vas conociendo a más personas que es mejor y vas trabajando con diferentes personas que a mí me parece muy bien”.(A1)

“Hemos aprendido de ellos, cada uno es distinto.”(A3)

"Me pareció una experiencia interesante por la permeabilidad que tiene el centro con el entorno."(M1)

Asimismo, esta experiencia piloto permitió tomar cuenta que contar con más voluntarios en el aula permitía atender mejor al alumnado con necesidades educativas como menciona la coordinadora de los grupos:

"Estoy pensando ahora en un niño, en un alumno que hasta ahora ha estado en pedagogía terapéutica, pero en grupos interactivos está hablando de alguna manera de igual a igual con las dos voluntarias que había, o sea, compartiendo tema con las dos voluntarias y con los otros críos, ¿no? $Y$ es un crío que siempre ha estado, o muchas veces, aislado... y cuando lo veo digo: pero míralo cómo está este chiquillo hoy. Sí, sí, sí.”(M2)

Fue en el segundo grupo de discusión cuando estas expectativas se delataron, y se puso en consideración la mejora de las relaciones entre los agentes educativos derivada de la participación en el centro de las familias, la comunidad local y la administración educativa. En definitiva, se transforma el vínculo entre los distintos agentes modificando el propio clima de la comunidad.

Estas relaciones mejoran a tres niveles, en un primer nivel la colaboración de las familias en el centro ha mejorado la relación de éstas con el profesorado:

"La relación del profesorado con los padres y madres no solo se da en el horario escolar sino que ha trascendido el mismo."(M1).

Este sentimiento se traslada también al hogar, donde padres e hijos comparten temas de conversación en casa sobre la escuela al sentirse parte integrante de la misma. Así lo relatan las familias:

"Antes no contaba nada de clase, y ahora nos dice lo que han hecho, con quién ha trabajado, que aporta al grupo.”(F1) 
“.. Y estoy viendo ahora desde dentro lo que se hace en la escuela, no sólo lo que me cuenta mi hijo, lo estoy viendo de primera mano y eso también es muy interesante, porque luego supone que a la hora de ayudar en casa tienes más referencias y no caes en contradicciones. Mi profesor me dice esto.... y tú me dices lo otro...." (F2)

Un alumno subrayó su entusiasmo ante la disposición de su padre como voluntario en el centro y explicaba:

"Me gusta que mi padre participe en mi cole, aunque no esté en mi aula, en casa me enseña cosas que enseña a otros niños. Él está muy contento de venir. También me gusta que haya padres de otros niños en mi clase porque así aprendemos mejor y es más divertido."(A1)

En un segundo nivel, la puesta en marcha de los grupos interactivos ha supuesto la apertura del centro al entorno educativo, produciéndose una mayor implicación de la comunidad local en el centro. Al respecto un padre señalaba:

"La gente del barrio conoce lo que pasa y cómo se trabaja en el colegio y quiere participar, y este proyecto camina gracias al voluntariado."(F3)

Además revierte en que todos los alumnos participan activamente gracias a la mediación del voluntario, personas cercanas a ellos, con quienes por ser del barrio, o de su entorno, crean un vínculo muy beneficioso y la comunidad educativa se fortalece de una forma activa y motivadora (Flecha, 2009).

Destacar aquí que casi la totalidad del voluntariado que no son familias procede de la asociación Ayuda Social Universitaria de La Rioja (ASUR), una organización que implica a los jóvenes estudiantes riojanos en proyectos de solidaridad social. Sus voluntarios proceden de las titulaciones de Trabajo Social, Enfermería, Magisterio, Filología Hispánica y Filología Inglesa. Desde la Universidad de La Rioja se les reconoce con un crédito el voluntariado realizado en el CEIP Caballero de la Rosa.

$\mathrm{Y}$ en un tercer nivel hablaríamos de influencia desde las administraciones, instituciones con las que en palabras del jefe de estudios tienen en la actualidad una relación más cercana, sobre todo con la Dirección General de Educación, que ha apoyado mucho el proyecto. Se amplió la jornada a una de las profesoras del centro otorgándole la responsabilidad de controlar y coordinar al voluntariado. En tiempo de crisis, el jefe de estudios presume de este logro al mismo tiempo que agradece la buena disposición de la Consejería, que con tales gestos, está apostando por modelos de escuela inclusivos.

"Tuvimos un momento de agobio y solicitamos que hubiera una persona dedicada a la coordinación de comunidades y tuvimos ahí una ampliación de personal. Es importante porque al principio, al ser algo nuevo, cuesta organizarlo y los voluntarios que si vienen que si no, que faltan, tienen que ir a buscar."(Je)

Por otra parte, como se ha adelantado anteriormente, cuando el centro comenzó a trabajar mediante grupos interactivos, y a contar con la participación de los agentes 
señalados se percataron que era la fórmula idónea para atender a los alumnos con necesidades educativas de apoyo educativo pues, al reducir la ratio de niños por un adulto, permitía la posibilidad de responder a sus dificultades de aprendizaje. Además, cabía la posibilidad de disponer de dos adultos por grupos y uno de ellos atender, personalmente, a las personas con necesidades de apoyo evitando que se ausente de la clase para recibir un apoyo adicional. El equipo directivo, al inicio, creyó conveniente contar con un adulto más en cada clase para paliar el vacío que podía ocasionarse ante posibles imprevistos surgidos a los voluntarios, dificultando así el buen funcionamiento. No obstante, en cualquier caso, repararon que ese voluntario adicional más en cada clase, podía apoyar también a aquellos alumnos con más dificultades rotando con ellos de actividad en actividad. En este sentido, el jefe de estudios y un padre manifestaron:

"La participación activa de los padres ayuda a crear una escuela inclusiva y nosotros lo estamos consiguiendo...”(Je)

"Sacar de clase a niños que no siguen el ritmo, es lo que se solía hacer y esa fórmula se llama exclusión. Comunidades propone justamente lo contrario, si en una clase hay alumnos que se van quedando atrás, pues quiere decir que se necesita más gente, y ahí está mi labor como voluntario. De lo que se trata es de incluir a universitarios, familiares, personas del barrio, para que haya más personal para atender a los alumnos."(F1)

"No obstante, los grupos interactivos implican un trasiego de personas en los pasillos de un colegio que debe ordenarse para evitar romper con el orden propio de un centro educativo. Así, a pesar de las ventajas, el hecho de aceptar la presencia de voluntarios en las aulas implica una planificación de horarios y grupos que resulta desconcertante en los inicios. “( $\mathrm{Je})$

Tanto en el primer grupo de discusión como en el segundo, los profesores manifestaron los problemas de coordinar horarios y voluntarios. En un curso académico lograron concentrar un total de 117 voluntarios y es complicado organizar una plantilla de tal envergadura así como sincronizar los horarios y su disponibilidad, más cuando no existe experiencia previa. En palabras del Jefe de Estudios:

"Algún inconveniente ha habido pero no lo catalogamos como tal. Estoy hablando del esfuerzo inicial que supone poner en marcha este proyecto. Yo como tutor, me ví un poco agobiado al principio porque es algo que no has tenido que preparar nunca. "(M1)

Entre líneas también se lee otro problema cuyo eco es esencial para el buen funcionamiento de los grupos interactivos, la responsabilidad de los voluntarios. Ellos deben de comprometerse fielmente a asistir puntualmente a sus llamadas como dinamizadores de grupos interactivos. Muchos eran alumnos universitarios quienes estaban sujetos también a posibles prácticas, exámenes y entregas de trabajos en sus estudios, o incidentes que pueden surgir. No obstante, en su mayoría avisaban al colegio vía mail o llamada telefónica pues el centro ha creado una red para avisar de cualquier incidencia. Esta problemática no queda contemplada en la literatura pero es 
de interés advertirla y proponer medidas en aquellos colegios interesados en reconsiderar estas propuestas.

Por otra parte, otro inconveniente, que no es una dificultad en sí misma sino una situación desconcertante, es dar voz a los alumnos y los padres e incluso a vecinos de barrio. Este hecho pude acarrear delegar funciones, escuchar opiniones contrarias o poner en entredicho ciertas decisiones. Exactamente no es un problema sino un efecto que puede "atormentar" a los profesores y en este sentido, en el segundo grupo de discusión un profesor aportó lo siguiente:

"Pudo resultar algo difícil entender que la clave del éxito iba a ser trabajar juntos con las familias y demás agentes educativos. Los docentes, igual que muchos gremios sentimos que tenemos una "autoridad". En este caso estás delegando parte de tu autoridad en tus chavales y tú puedes pensar igual o diferente, y te enfrentas a ese diálogo enriquecedor pero entiendo que alguno profes no quieran enfrentarse, aunque en realidad lo que tienen es miedo."(M2)

El jefe de estudios y un padre también aportaron su opinión sobre este tema:

"Yo me he pegado auténticas "bajadas de la tarima". Los profesores tenemos que ser conscientes ahora de que con este proyecto ninguna voz vale más que otra."(Je)

\section{Discusión y conclusiones}

En el apartado de resultados se han vislumbrado las impresiones de padres, profesores y alumnos sobre la experiencia incipiente del Colegio Caballero de la Rosa mediante grupos interactivos con el objeto de analizar su alcance en el rendimiento académico, participación y convivencia entre el alumnado.

A través de dos grupos de discusión, se ha pretendido cotejar su logro en sus primeros pasos haciendo uso de esta metodología en el curso escolar 2012/2013 tras una experiencia piloto. A la luz de los resultados, es arriesgado concluir, y medir, que los grupos interactivos y otras prácticas han logrado mejorar los resultados de aprendizaje pero sí pueden intuirse visos de una mayor implicación del alumnado y, por ende, un creciente compromiso con la tarea motivado por la dinámica de la misma tal y como lo avalan estudios de carácter internacional que demuestran que la implicación de familiares y escuela mejoran el fracaso escolar, sobre todo de minorías culturales (Appel y Beane, 2000; Jaussi y Luna, 2002; Flecha, 2009; Flecha y Soler, 2013). Además, permite un aprendizaje con los otros a través de las interacciones generadas durante el desarrollo de los grupos interactivos tomando cuenta de los logros alcanzados porcada alumno. El ámbito convivencial también requiere de más tiempo para recoger sus frutos pues, para ello, es prioritario establecer lazos de colaboración del centro con la familia y la comunidad educativa (Dearing, Kreider, Simpkins, y Weiss, 2006; Flecha, 2009; García y Villar, 2011). Esto es, asentada la participación de todos los agentes implicados y logrados vínculos de colaboración, puede concluirse que la convivencia mejora (Flecha, 2009; Oliver, de Botton, Soler y 
Merril, 2011). En este sentido, puede decirse que el colegio está bien encauzado pues la participación en sus distintos planos ha sido constatada en este primer curso en base a las impresiones de unos y otros como ha podido leerse en los testimonios aquí presentados. Así, los participantes parecen reconocer múltiples ventajas pero, sobre todo, resaltaron dos: ahora los padres pueden ser conscientes en primera persona de lo que se vive en las aulas y, gracias a esa participación se puede atender a todos los alumnos, especialmente aquellos más vulnerables (Valls y Kyriakides, 2013) sin tener que excluirles fuera de las aulas y sustituir la "segregación por la inclusión igualitaria" (Aubert, García y Racionero, 2009, p. 134).

De otra parte, otra conclusión reseñable es la responsabilidad compartida de la función docente que, por el contrario, se ha caracterizado por una labor aislada, los profesores manejan el timón en clase y cualquier visita externa se considera intrusión. Los participantes en el grupo de discusión manifestaron que lo más dificultoso de interiorizar ha sido delegar la autoridad del maestro a padres, alumnos y personal no docente (Aubert, García y Racionero, 2009). Sin embargo, el objetivo no es restar autoridad sino ofrecer nuevas perspectivas, completar información y aprender del diálogo en igualdad de condiciones (Aubert, Flecha, García, Flecha y Racionero, 2008; Flecha, 2009). En palabras de Aubert, García y Racionero (2009, p.131) a través del diálogo "se rompen las relaciones jerárquicas y autoritarias en los centros educativos”. Los profesionales no son los meros transmisores de información y por ello, es necesario que los profesores lleven una acción armonizada con las demás entidades transmisoras de conocimientos con el fin de desarrollar la tarea educativa de forma provechosa. "Si la escuela ya no es la única y principal fuente de conocimientos, la influencia de todas estas interacciones tiene un gran potencial cuando apuntan en la misma dirección” (Flecha, 2006, p.64).

Por ello, lejos de intrusismo, la posibilidad de contar con más personas en el aula ofrece lentes nuevas que pueden aportar mejoras, ofrecer ayuda y, sobre todo, dar más luz al aprendizaje de los alumnos así como otros modelos de trabajo y conducta (Elboj y Niemela, 2010). Como indican Flecha, García, Gómez y Latorre (2009, p.163), "se trata de personas con perfiles muy diferentes al del profesorado y que, algo que es muy importante, tienen imágenes diferentes del alumnado desde las que es posible transformar las interacciones educativas". Los grupos interactivos postulan el valor didáctico de aprender mediante el diálogo y la interacción entre iguales considerando a todas las personas en condición de aportar algo. Así, los adultos brindan la oportunidad de abrir ese diálogo, aprender en grupo e incentivar la participación de todos los alumnos alentando a aquellos que tienen menos posibilidades. No es una estrategia de compensación ni de refuerzo, es una llave para la aceleración del aprendizaje. Su engranaje requiere planificación, organización y coordinación, este puede ser el principal inconveniente. No obstante, se subsana con la voluntad e interés de los implicados y con la ayuda de la comunidad educativa. La disposición de los padres, el empuje de la Administración Educativa y la colaboración de asociaciones como Asur han contribuido a lograr que el Caballero de la Rosa sea la primera comunidad de Aprendizaje en la ciudad de Logroño. Es un ejemplo de escuela inclusiva pues ha abierto sus puertas al barrio siendo un referente de sintonía y trabajo conjunto entre familia y escuela. Así puede decirse que la educación inclusiva exige 
del trabajo colaborativo y dialogado de todos, y la actitud favorable del profesorado es la clave para lograrlo. No obstante, estos resultados están sujetos a limitaciones del estudio como el hecho de que se infieren de una única experiencia que, a su vez, pone en duda la representatividad de la muestra. Sin embargo, no es pretensión extrapolar resultados sino reflexionar sobre propuestas metodológicas de enorme calado en la actualidad que persiguen el logro de ambientes educativos más inclusivos.

Las Comunidades de Aprendizaje parten de los sueños de padres, profesorado y alumnado. En este caso, el sueño se ha cumplido: son ya una comunidad de aprendizaje. Son comunidad en cuanto que aúnan esfuerzos por lograr el desarrollo armónico de todos ellos y de aprendizaje pues son muchos los aprendizajes ya adquiridos, al margen del rendimiento académico. Han aprendido el valor del barrio y a apreciar el trabajo contando con las voces de todos. Un sueño hecho realidad pero que exige de tesón para lograr el mejor de los sueños: contagiar al resto con el ejemplo y lograr una comunidad de comunidades con otros colegios.

\section{Referencias bibliográficas}

AUBERT, A., FLECHA, A., GARCÍA, C., FLECHA, R. Y RACIONERO, S. (2008). Aprendizaje dialógico en la Sociedad de la Información. Barcelona: Hipatia.

AUBERT, A., GARCÍA, C. Y RACIONERO, S. (2009). El aprendizaje dialógico. Cultura y Educación. 21 (2), 129-129.

APPEL, M.V. Y BEANE, J.A. (2000). Escuelas democráticas. Madrid: Morata.

CRESWELL, J.W. Y MILLER, D.L. (2000). Determining validity in qualitative inquiry.Theory into Practice, 39, (3), 124-131.

DEARING, E., KREIDER, H., SIMPKINS, S., Y WEISS, H. B. (2006). Family involvement in school and low-income children's literacy: Longitudinal associations between and within families. Journal of EducationalPsychology, 98, 653-664.

ECHEITA, G. Y AINSCOW, M. (2011). La educación inclusiva como derecho. Marco de referencia y pautas de acción para el desarrollo de una revolución pendiente. Tejuelo, 12, 26-46.

ELBOJ SASO, C., PUIGDELLIVOL AIGUADÉ, I., SOLER GALLART, M. Y VALLS CAROL, R. (2002). Comunidades de aprendizaje. Transformar la educación. Barcelona: Graó.

ELBOJ, C., PUIGDELLIVOL, I., SOLER, M. Y VALLS, R. (2005). Comunidades de aprendizaje. Barcelona: Graó.

ELBOJ, C. Y NIEMELLA, R. (2010). Sub-communities of mutual learners in the classroom: the case of interactive group. Revista de Psicodidáctica, 15(2), 177-189. 
FLECHA, R. Y SOLER, M. (2013).Turning difficulties into possibilities: engaging Roma families and students in school through dialogic learning.Cambridge Journal of Education, 43(4), 451-465.

FLECHA, R. Y ORTEGA, S. (2012). Comunidades de aprendizaje . Educadores: Revista de renovación pedagógica, 243, 8-21.

FLECHA, R. (2006). La participación de las familias en las comunidades de aprendizaje, en Liarte, C. (Coord.) Participación de las familias en la vida escolar: acciones y estrategias (pp. 59-68). Ministerio de Educación y Ciencia, Secretaría General de Educación.

FLECHA, R. (2009). Cambio, inclusión y calidad en las comunidades de aprendizaje. Cultura y Educación, 21 (1), 157-159.

FLECHA, R., GARCÍA, R., GÓMEZ, A. Y LATORRE, A. (2009). Participación en escuelas de éxito. Una investigación comunicativa del proyecto INCLUD-ED. Cultura y Educación, 21 (2), 183-196.

GALLARDO, P. Y CAMACHO, J.M. (2008). La investigación participativa y su aplicación en el campo social y educativo. Sevilla: Wanceulen Educación.

GARCÍA, A. Y VILLAR, C. (2011). La aportación del proyecto de comunidades de aprendizaje a la transformación social y educativa de un barrio. Tendencias Pedagógicas, 18, 207-232.

INCLUD-ED CONSORTIUM. (2009). Actions for sucess in schools in Europe. Brussels: European Commision.

JAUSSI, M. L. Y LUNA, F. (2002). Experiencias de éxito. Cuadernos de Pedagogía, 316, 39-67.

KRUEGER, R. (1991). El grupo de discusión. Guía práctica de la investigación aplicada.Madrid: Pirámide.

KRUEGER, R. Y CASEY, M. (2000). Focus Groups. A practical guide for applied research. Thousand Oaks, CA, Sage Publications, Inc.

LLOPIS, R. L. (2004).Grupos de discusión. Madrid: Esic Editorial.

LÓPEZ DECEBALLOS, P. (1998). Un método para la investigación-acción participativa. Madrid: Editorial Popular.

MARTÍNEZ, R. A. (2007). La investigación en la práctica educativa: Guía metodológica de investigación para el diagnóstico y evaluación en los centros docentes. Madrid: Centro de Investigación y Documentación Educativa.

OLIVER, E., DE BOTTON, L., SOLER, M. Y MERRIL, B. (2011). Culture Intelligence to Overcome Educational. Exclusion Qualitative Inquiry, 17 (3), 267276.

RACIONERO, S. Y VALLS, R. (2007). Dialogic learning: A communicative approach to teaching and learning. En J. Kincheloe \& R. Horn (Eds.), The praeger 
handbook of education and psychology (pp. 548-557). Wesport, Connecticut: Greenwood Publishers.

RACIONERO, S. Y SERRADELL, O. (2005). Antecedentes de las Comunidades de Aprendizaje. Educar, 35, 29-39. http://colaboraeducacion.juntadeandalucia.es/ educacion/colabora/web/guest/home(Consultado 20 de enero de 2014)

SALAZAR, M. C. (1992). La investigación acción participativa, inicios y desarrollos. Madrid: Editorial Popular.

SUÁREZ, C., DEL MORAL, G. Y GONZÁLEZ, M. T. (2013). Consejos prácticos para escribir un artículo cualitativo publicable en psicología. Psychosocial Intervention, 22, 71-79.

VALLS, R. Y KYRIAKIDES, L. (2013). The power of Interactive Groups: how diversity of adults volunteering in classroom groups can promote inclusion and success for children of vulnerable minority ethnic populations. Cambridge Journal of Education, 43, 1, 17-33.

\section{Correspondencia con las autoras}

Edurne CHOCARRO DE LUIS

Edificio Vives

Departamento de Educación

Universidad de La Rioja

C/ Luis de Ulloa, $\mathrm{s} / \mathrm{n}$.

26004 Logroño.

e-mail: edurne.chocarro@unirioja.es

Magdalena SÁENZ DE JUBERA OCÓN

Edificio Vives

Departamento de Educación

Universidad de La Rioja

C/ Luis de Ulloa, $\mathrm{s} / \mathrm{n}$.

26004 Logroño.

e-mail: m-magdalena.saenz-de-jubera@unirioja.es 
